\section{P629 NEONATAL SUBCUTANEOUS FAT NECROSIS A FIVE CASES STUDY}

Manel Charfi*, Sahar Ben Amar, Amel Ben Hamad, Chiraz Regaieg, Amira Bouroui, Ridha Regaieg, Nedia Hmida, Afef Ben Thabet, Abdellatif Gargouri. Department of Neonatology, Hedi Chaker University Hospital, Sfax, Tunisia

\subsection{6/archdischild-2019-epa.960}

Introduction Neonatal subcutaneous fat necrosis is a rare condition. It's due to a transient panniculitis and present as an erythematous nodules and indurate plaques over bony prominences that appears in the first weeks of life. We aim to identify the risk factors, clinical aspects, and outcomes of this rare condition.

Methods It's a retrospective study including five cases of subcutaneous fat necrosis hospitalized in the neonatal intensive care unit of Sfax between 2016 and 2019.

Results Two boys and three girls were registered. Only one newborn was a preterm of 35 weeks of amenorrhea. Two mothers had gestational diabetes and the others had uncomplicated pregnancies. Four newborns presented birth asphyxia requiring resuscitation in the delivery room. The median 1 5 minutes Apgar scores were respectively 5.8 - 7.5. Three newborns were macrocosmic. The others had a normal birth weight. Subcutaneous fat necrosis lesions were noticed by the two first weeks of life in all cases with a mean of 7.4 days (4 to 18 days).All newborns had diffuse skin lesions. It was meanly localized on the sacro-coccygeal region, back, shoulders and limb roots. All skin lesions resolved spontaneously within two to three months except for one newborn who presented a liquefaction of the content of one of his lesions by the day 11 of life. It was a median lesion measuring $6 * 10 \mathrm{~cm}$ localized in the back. Monitoring and laboratory analysis showed thrombocytopenia that preceded the onset of the lesions in all cases. It was severe in 3 cases with an average of 40000 (27 000 to 58 000). Three newborns developed hypercalcemia. It was severe for only one newborn with a serum level reaching $4.42 \mathrm{mmol} / \mathrm{L}$ by day 30 of life. Follow up showed associated nephrocalcinosis in two cases and metastatic skin calcifications in one case. For the newborn with severe hypercalcemia, as initial treatment he received low calcium and vitamin D formula, hyperhydration, intravenous methylprednisolone and furosemide. Due to a lack of response, we started treatment with pamidronate with a favorable evolution. Normalization of the serum calcium level as well as the renal ultrasound was finally obtained by the age of 4 months.

Conclusion Subcutaneous fat necrosis is a self-limited condition with a good prognosis generally. However, it may be associated to rare but serious complications especially thrombocytopenia and hypercalcemia.A long term clinical and biological follow-up for the possible onset of these complications is then necessary.

\section{P630 NON INFECTIOUS COMPLICATIONS RELATED TO CENTRAL CATHETER IN NEONATES (A STUDY OF 8 CASES)}

Manel Charfi*, Amel Ben Hamad, Sahar Ben Amar, Chiraz Regaieg, Amira Bouraoui, Ridha Regaieg, Nedia Hmida, Afef Ben Thabet, Gargouri Abdellatif. Department of Neonatology, Hedi Chaker University Hospital, Sfax, Tunisia

10.1136/archdischild-2019-epa.961
Introduction The use of central catheters in the neonatal period is essential for the management of neonates with vital distress or premature newborns. Nevertheless it can be associated to serious complications specially related to leakage of parenteral nutrition.

Methods It's a retrospective study of all cases of non infectious complication related to central catheter registered in the neonatal intensive care unit of Sfax between 2012 and 2018.

Results We registered 5 cases of pericardial effusion, one case of pleural effusion, one case of ascites and one case of pneumopericardium. All newborns were premature (29 to 33 weeks of amenorrhea). Five patients had epicutaneo-cave catheters, two patients had umbilical venous catheters and one had broviac catheter. The catheter position was intracardiac in all cases. The incident occurred in an average of 2.75 days after the catheter insertion (1to 6 days). Clinical manifestations were no specific miming sepsis in almost all cases. Hemodynamic disorders with tachycardia, tachypnea and skin mottling were noticed in 4 cases of pericardial effusion. Three preterm newborns with tamponade presented moreover severe apnea. The newborn with ascites presented abdominal distension and tachypnea. Diagnosis was made by ultrasound exam in all cases related to leakage of parenteral nutrition. For the case of pneumopericardium diagnosis was made on chest radiography. Management consistent on oxygen administration associated to hemodynamic support all cases. An ultrasoundguide pericardium puncture was performed in four cases of pericardial effusion. The catheter was removed in five cases and repositioned in three cases. Outcome was favorable four all newborns.

Conclusion Clinician should be aware of the risk of leakage of parenteral nutrition. Thus every neonate with central catheter must be monitored and the extracardiac position of the catheter must be systematically verified.

\section{P631 SCORE FOR NEONATAL ACUTE PHYSIOLOGY PERINATAL EXTENSION II (SNAPPE II) IN NEONATES WITH ACUTE KIDNEY INJURY}

Silvana Naunova Timovska*, Aspazija Sofijanova, Mica Kimovska, Tamara Voinovska, Hristina Mandzukovska, Spasija Neskova, Danilo Nonkulovski, Olivera Jordanova, Vladimir Timovski. University Children Hospital, Skopje, Macedonia, the former Yugoslav Republic of Macedonia

\subsection{6/archdischild-2019-epa.962}

Background Acute kidney injury is a serious clinical problem in neonatal intensive care unit. It is defined as a sudden decrease in kidney function resulting in derangements in fluid balance, electrolytes, and waste products. SNAPPE 2 score is a useful tool for assessing the severity of the disease that correlates with neonatal mortality.

Objective The aim of the study was to determine the incidence of AKI and the role of SNPPE 2 score in predicting mortality and morbidity in AKI in neonates.

Methods The study was designed as a prospective, clinical, epidemiological investigation conducted in the period of three years, which included 100 newborn infants $(50$ with AKI and 50 without AKI) hospitalized in NICU of University Children's Hospital. The severity of the illness of hospitalized newborn infants was estimated with SNAPPE 2 score realized in the first 12 hours of admission in NICU. Medical data records of admitted neonates with AKI were analyzed. The material was statistically processed using methods of descriptive statistics. 